\title{
Battery Management System Integrated with CAN BUS Safety Control Environment for Electric Vehicle
}

\author{
S. Divyashree ${ }^{1}$ \\ ${ }^{1}$ Department of Electronics and Communication \\ Engineering, \\ SRM Institute of Science and Technology, \\ Kattankulathur, Chennai
}

\author{
Prassanna Madhavan ${ }^{2}$, A.Ranjeev ${ }^{3}$ \\ ${ }^{2,3}$ Department of Electrical and Electronics Engineering, \\ SRM Institute of Science and Technology, \\ Kattankulathur, Chennai
}

\begin{abstract}
Battery Management System (BMS) is one of the most crucial and essential components of an Electric Vehicle. The main feature of a BMS is to safeguard the battery and make the operation reliable and smooth. To achieve the same, Battery State of Charge (SoC) evaluation, Charge Control and Cell Balancing have to be implemented in the BMS. BMS performs many complex operations that require constant optimization of the BMS for the improvement of battery life cycle, engine performance, energy enhancement and security of the vehicle. The need for security increases with the increase in the control units that favor data theft and security of the vehicle. A Controlled Area Network (CAN) based communication control system fulfills the need of a closed environment security system that optimizes the BMS and minimizes cyber threat. The main objective of this study is to give a detailed analysis of two key components of an electric vehicle viz., the BMS and the CAN bus that is used for communication. This study also covers the general model circuitry diagrams that are used to integrate the BMS and CAN bus that can be implemented in the real world. This work also hopes to help the readers who are interested in pursuing work in this field of vehicular design.
\end{abstract}

\section{INTRODUCTION}

Years of fossil fuel usage has paved way for major environmental catastrophes. Public and experts all around the world are moving towards a path of lessening this problem. Global warming is primarily caused by the emission of $\mathrm{CO}_{2}$ that is let out by the burning and combustion of fossil fuels for generation of power for domestic and industrial purposes. By producing energy from renewable energy sources, we reduce Carbon emissions and thereby reduce the future carbon footprint. Thanks to this global surge of $\mathrm{CO}_{2}$ emission, the production of electric vehicles is increasing at a rapid pace. The governments all around the world are adapting to this new change and are implementing action plans to create awareness amongst the citizens of the country. This encouragement drives the people to invest in Electric Vehicle. In the years to come the world will primarily run on full electric vehicle that make use of battery and an electric motor for its operation. As this happens there would be very less need for petroleum, diesel and other fossil based fuels. Fig. 1 shows a basic schematic of a power system that is implemented in an Electric Vehicle and from the diagram we can infer that the Battery Management System plays a crucial and essential role in the smooth working of the EV power system. The BMS is mainly composed of battery packs, electronic control units and numerous sensors used for various applications. A battery pack consists of a number of cells arranged in a series - parallel combination. The number of cells in series may vary according to the overall voltage requirement. The more the number of cells in series, the higher the voltage and the current increases with the increase in cells in parallel.

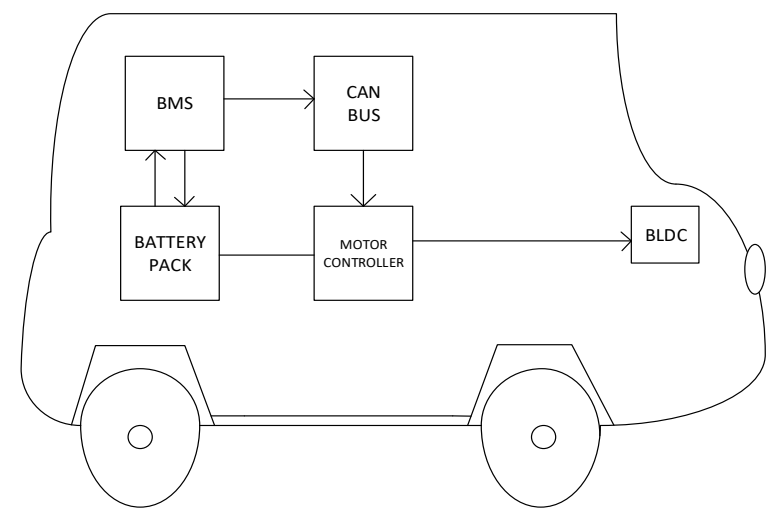

Fig.1 Basic Overview of the system

The BMS is used to fulfill the purpose of monitoring the battery packs. It monitors a range of parameters including the battery current, voltage, remaining travel range of the battery, State of Charge (SoC), State of Functioning (SoF) of a particular function or task and State of Health $(\mathrm{SoH})$. In the upcoming version of BMS in EV, it is said that the system will estimate the State of Life (SoL) of the battery pack that will calculate the total life expectancy under normal working environment. The State of Charge of a battery is theoretically defined as the ratio of available battery capacity to the maximum capacity of the battery pack. In general, it is given as the amount of charge that is available in the cell. The work done by J. Kim et al is shown to estimate the battery SOC using a charge distributer or equalizer which uses the open circuit voltage of the battery pack [1]. The $\mathrm{SoH}$ is defined as the maximum capacity of the battery pack that is determined by the number of charge and discharge cycles of the pack. It is one of key determining factors of battery performance and forms the crux of battery life estimation. The Controlled Area Network (CAN) is one of the most preferred communication protocols in modern 
vehicles. It is used for the communication between numerous control units and the BMS. In normal cases, the communication between the battery pack and the control units is done by wiring, which becomes bulky and does not allow for adding of extra components. In order to solve this problem, the implementation of CAN bus-based communication is essential as it reduces the wiring required between the control units. It uses a single serial bus which combines all the wiring into a single node using the controlled area network protocol. The modern automotive companies look to install in vehicles a distributed architecture as this reduces the complexity and chances of failure [2].The distributed architecture allows room for more flexibility and expansion when compared to a centralized network architecture [3]. The CAN control system is very transparent in nature. Instantaneous message signals such as the speed of the motor or the battery pack temperature are not communicated to one particular node but to the entire system of control units [4]. The work done in this paper gives an outline of a CAN bus integrated BMS that will explain in detail about each system and the integrated system of CAN and BMS. A range of existing ideas are put together for detailed future researches.

\section{BATTERY MANAGEMENT SYSTEM (BMS)}

Battery Management System is a structure that controls two units which uses communication protocols to monitor each battery pack and ensure the inter cell balancing operation takes place optimally. It collects the data and checks whether it matches the safety standards to ensure security. It measures various parameters such as cell balancing, charging infrastructure and charge and discharge rate.

\section{i) BMS architecture}

The Architecture of the Battery Management System consists of two main divisions such as predicting accurate SoC (State of Charge) and SoH (State of Health). Although, the SoC determines the charging cycles for regenerative braking, in case of high power discharge, together the SoC and $\mathrm{SoH}$ control the operations that determine the heat and thermal management, diagnosis of fault due to shutdown, the power quality, cell degradation, optimal cell charging patterns and balanced battery cell life. The coulomb counting method is widely used for current integration to calculate the SoC. The battery readings are noted to calculate the SoC defined as,

$$
Q(t)=\frac{Q\left(t_{T}\right)_{\text {remaining capacity }}}{Q_{\text {overall capacity }}}
$$

Where, $\mathrm{Q}(\mathrm{t})$ is the total SoC and $\mathrm{Q}\left(\mathrm{t}_{\mathrm{T}}\right)$ is defined as total remaining capacity. The SoC can alternatively also be found by,

$$
Q(t)=Q\left(t_{0}\right)+\frac{1}{C_{\text {estimated }}} \int_{t_{0}}^{t+\tau} I_{B A T T E R Y} \times I_{\text {LOSS }} d t
$$

Where, $\mathrm{C}_{\text {estimated }}$ is the estimated capacitance, $\mathrm{I}_{\mathrm{BATTERY}}$ is the total battery current along with $\mathrm{I}_{\text {LOSs }}$ being the loss current. $\mathrm{Q}\left(\mathrm{t}_{0}\right)$ is the initial SoC observed. The total remaining capacity $\mathrm{Q}\left(\mathrm{t}_{\mathrm{T}}\right)$ can be deduced as

$$
Q\left(t_{T}\right)=Q\left(t_{0}\right)-\int_{0}^{t_{T}} I_{\text {BATTERY }}(t) d t
$$

For SOH operation,

$$
Q_{\text {overall capacity }}=Q_{\text {nominal capacity }} \times S O H
$$

The formula given above is used to determine the cell balancing state, based on the current and voltage data in the battery module. The practical way to implement this efficiently is to divide the system into master and slaves subsystems. This system is implemented using a star topology pattern that lets the master to control the current flow using the voltage and temperature data while multiple slaves can be connected to a single master for initiating battery protection. The Master unit is mainly responsible for the Power Distribution, Communication, and State estimation. The slave is mainly connected only for the purpose of monitoring and cell balancing.

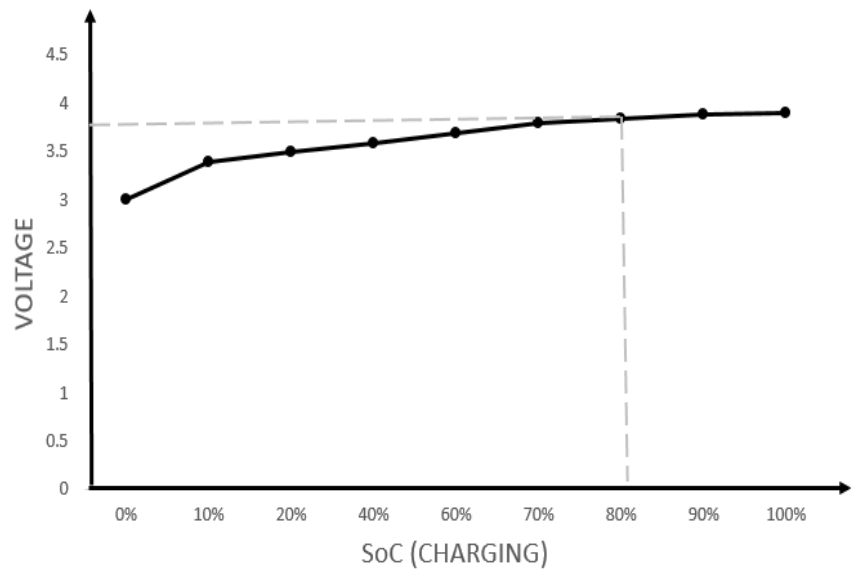

Fig.2.a.Voltage v/s charging SoC

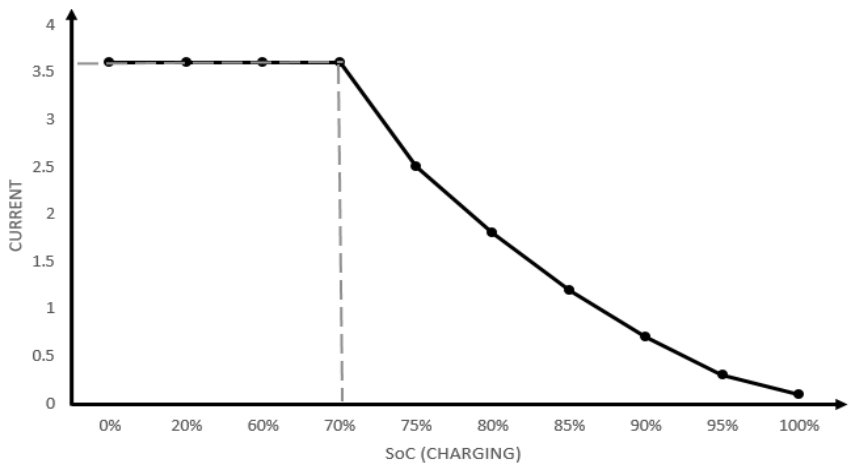

Fig.2.b. Current v/s charging SoC

\begin{tabular}{|c|c|c|}
\hline SoC & CV & CC \\
\hline $0 \%$ & 3.09 & 3.69 \\
\hline $10 \%$ & 3.29 & 3.69 \\
\hline $20 \%$ & 3.41 & 3.68 \\
\hline $30 \%$ & 3.55 & 3.68 \\
\hline $40 \%$ & 3.59 & 3.69 \\
\hline $50 \%$ & 3.62 & 3.68 \\
\hline $60 \%$ & 3.69 & 3.67 \\
\hline $70 \%$ & 3.85 & 3.57 \\
\hline $80 \%$ & 3.86 & 1.95 \\
\hline $90 \%$ & 3.89 & 0.87 \\
\hline $100 \%$ & 3.91 & 0.05 \\
\hline
\end{tabular}

Table 1.a. Results of Charging SoC 
The graph in Fig 2.a. and Fig 2.b. show the charging infrastructure based on the electrical potential of the cell. This is determined by using a circuit voltage test with a negligible load connected to see the battery as shown in Table 1.a. The charging is based on two stages Constant Current (CC) and Constant Voltage (CV). CC brings the charge to $70-75 \%$ approximately and then it enters the $\mathrm{CV}$ stage where it reaches the saturation point. After the saturation point the voltage is constant while the current drops to $0.05 \mathrm{C}$.

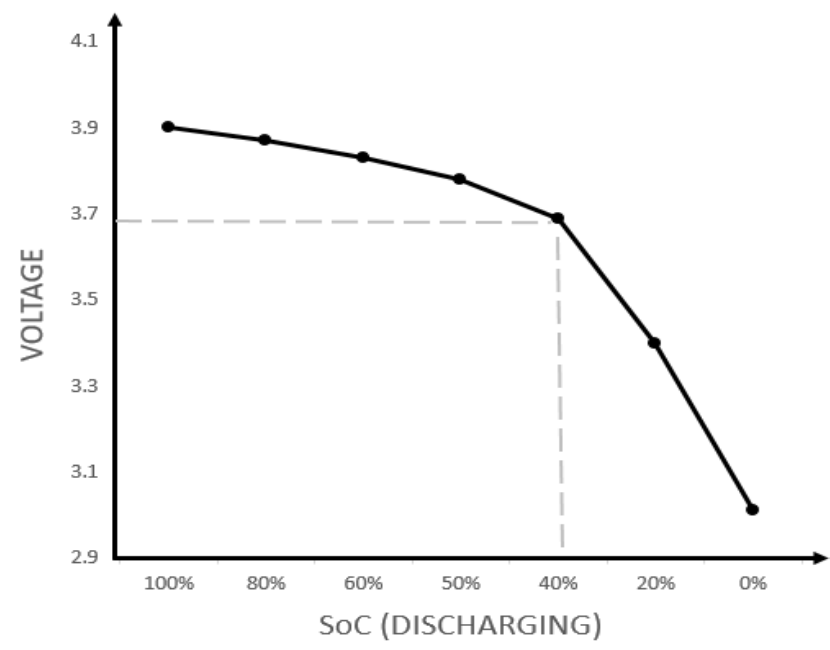

Fig 2.c. Voltage v/s discharging SoC

\begin{tabular}{|c|c|}
\hline SoC & DISCHARGE \\
\hline $100 \%$ & 3.09 \\
\hline $90 \%$ & 3.29 \\
\hline $80 \%$ & 3.41 \\
\hline $70 \%$ & 3.55 \\
\hline $60 \%$ & 3.59 \\
\hline $50 \%$ & 3.62 \\
\hline $40 \%$ & 3.69 \\
\hline $30 \%$ & 3.85 \\
\hline $20 \%$ & 3.86 \\
\hline $10 \%$ & 3.89 \\
\hline $0 \%$ & 3.91 \\
\hline
\end{tabular}

Table 1.b. Results of Discharging SoC

Fig.2.c shows the discharging capability of a cell considering that the cell has a life of 250-450 charge cycles. The reading is noted based on the discharge rate for every charge and discharge cycle. The discharge graph shows from fully charge to fully discharge. If there is a high discharge rate, the cell may lose its properties due to internal resistance.

\section{ii) Electric Circuitry}

The circuit drawings in the BMS plays a pivotal role for designing the whole unit in a car. It is illustrated and designed in a way to make the understanding of the circuitry easily. The main systems in a BMS consists of a Pedal Assembly Subsystem, Battery Pack Subsystem, Motor Controller Subsystem and Dash Board Subsystem. These subsystems consist of multiple sensors that detect errors and give feedback via CAN Bus. The battery cells are stacked over one another to form a battery pack which is the main power generation unit of the electric vehicle. The battery pack needs to be constantly monitored for short circuits, over voltage, over current, etc. and this is where the BMS comes in to play. It not only does the function of monitoring of the battery pack parameters but it also houses the battery pack along with the parameter sensor to form a closed environment.. The BMS forms an intricate system which allows the battery pack to power the motor control system via the discharge circuit. The BMS also connects itself to the current sensor that detects the amount of current incoming from the $\mathrm{AC}$ power outlet. It houses a small efficient rectification unit that converts the $\mathrm{AC}$ power to $\mathrm{DC}$ and charges the battery. The BMS also makes use of a cooling system needed to maintain an optimum working environment temperature for the battery pack and regulates the temperature by using the fan control and fan monitoring unit. The voltage, current and other pack parameters are tapped with the cell tapping unit. These parameters are then digitalized and sent to the data acquisition unit where logging of key information happens. The battery parameters from the BMS are transmitted to the motor system through the CAN bus. The basic model for the simple understanding of BMS power and signal transmission is described in the circuitry below:

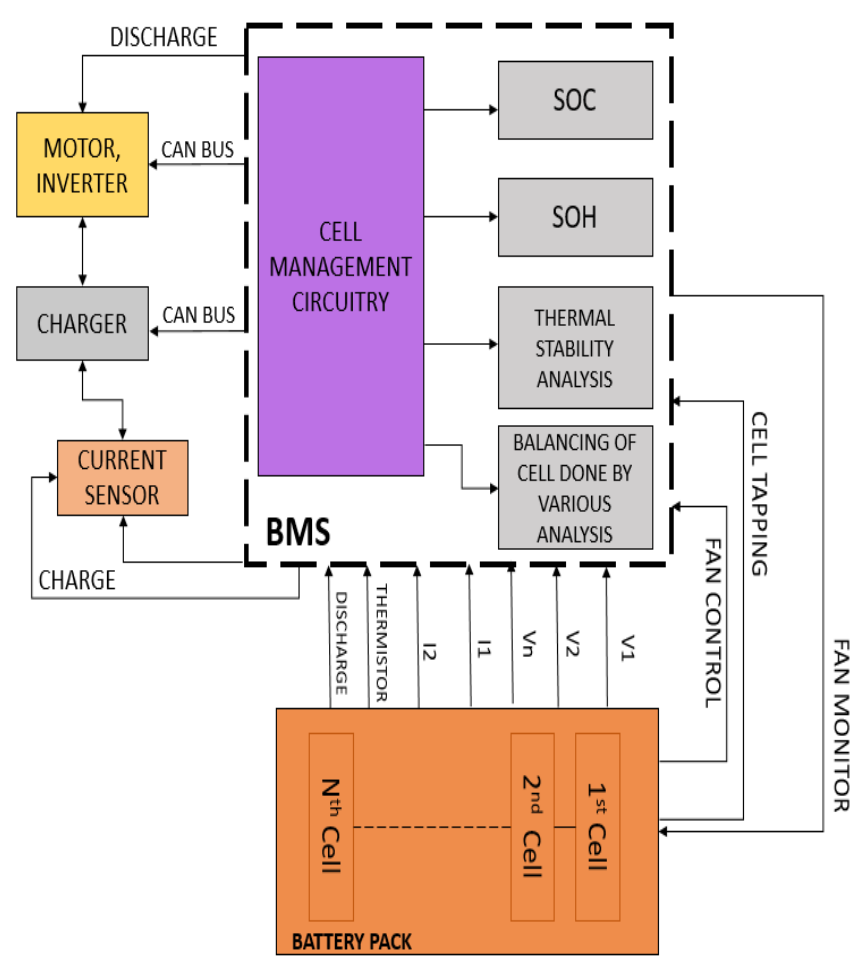

Fig.3 Battery Management System in EV

\section{CONTROLLER AREA NETWORK (CAN) BUS AND HIGH VOLTAGE POWER SYSTEM}

The Controlled Area Network (CAN) is one of the most well-structured communication protocols for a serial bus communication system. The CAN Bus system is used to connect multiple control systems in a single large network. It uses multiple master nodes to direct various slave nodes. 
The CAN Bus is based on messages and nodes of the network that do not require specific addresses. The node to which the information has to be conveyed will have the address of the message being transmitted and thereby intimating the priority to the system. The nodes do not have a specific number and can be changed at any instance without disturbing the communication of the rest of the system. The main role of the CAN Bus is to act as the safety control lead of the entire vehicle. It is not only responsible for the safety control but also the working of automation in various parts of the car. It has to manage the working and functioning of all the voltage components of the EV and also notify the driver in case of a surge, short circuit or failure. In Fig.4, the basic layout of the high power high voltage system of an EV is given. The CAN bus will act as the connecting control system for the entire high voltage system. The battery pack as shown in Fig. 4 is the main power supply unit for the motor drive system. It is protected by means of a high voltage switch or a high voltage disconnect and a fuse. The DC-DC converter is used to bring down the voltage. In case of voltage over shoot, the high voltage disconnect switch opens in advance when it detects a peak overshoot by means of the ECUs which disconnect the system and open the Ground Low Voltage System (GLVS). The system as whole is divided into different sub systems such as the motor controller sub system, dash board sub system and the battery pack sub system, each of them having a safety system installed in it. The main need for a precharge and discharge relay is to protect the motor control unit from random and sudden spike voltages.

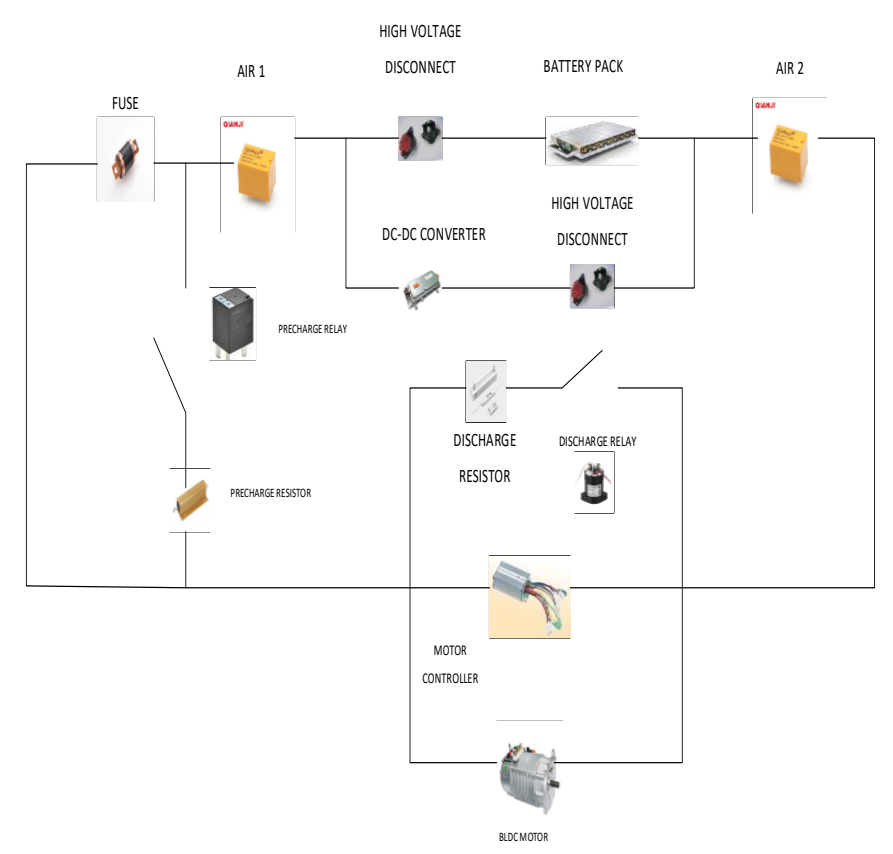

Fig.4 High Voltage system

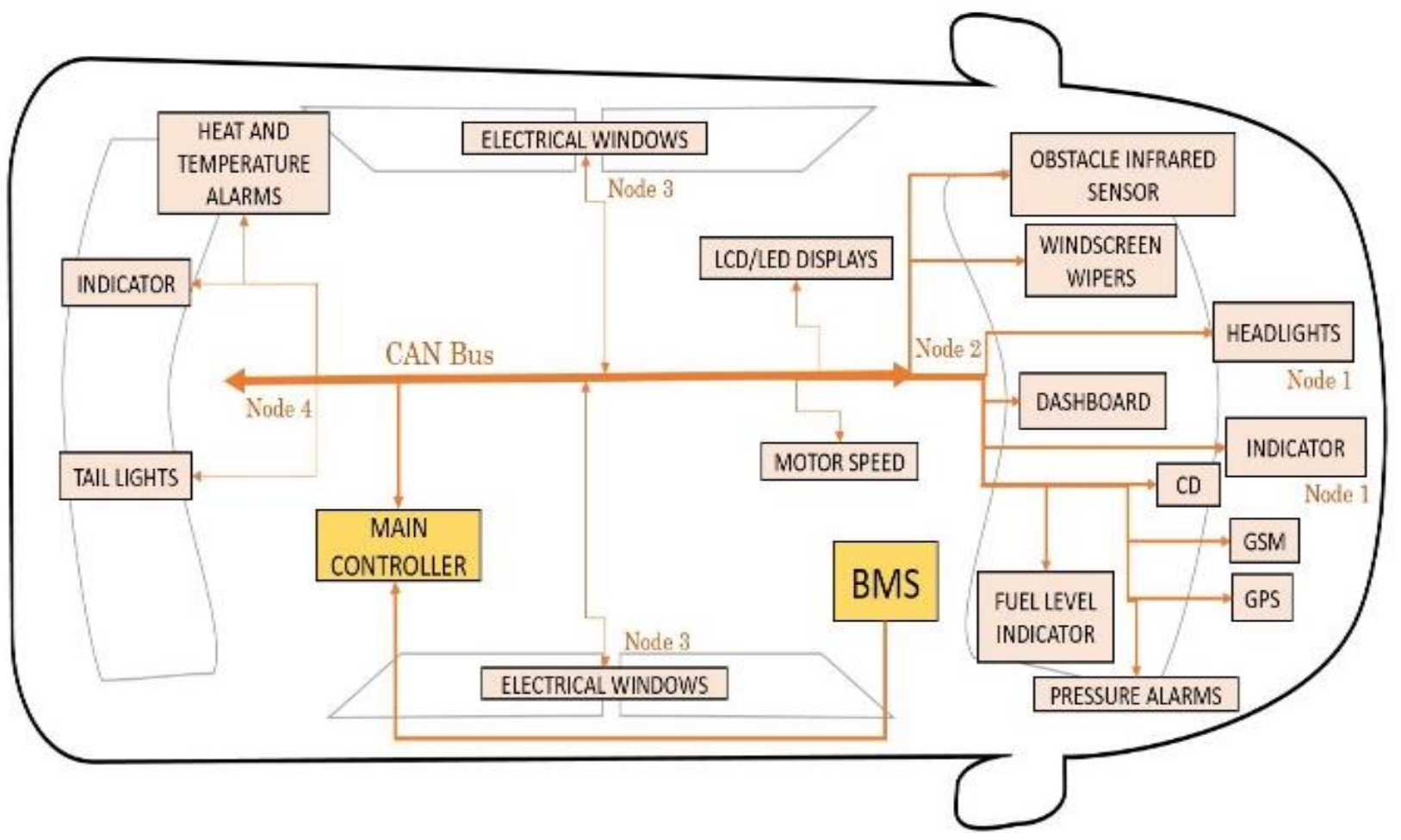

Fig.5 CAN Bus Control System in Electric Vehicle

There are multiple sensors in and around the HVS such as the torque encoders which vary the resistances with the help of potentiometer boxes to create a constant torque which is based on a constant current at the back end. In addition there are thermal sensors, current sensors, voltage sensors, proximity sensors and speed sensors. The CAN bus plays a dual role by being the primary safety messenger and the master communication control unit for all the Electronic Control Units (ECU) as shown in Fig.5. So all the sensors connected to different control units have to be in contact 
with the CAN. The CAN not only keeps contact with the electronic control units across the vehicle but also with the various subsystems in the vehicle like the Advanced Antilock Braking System (AABS) and the Battery Management System. The system where the BMS integrates with the CAN bus will be discussed in detail in section 4 .

\section{- THE BATTERY SUBSYSTEM}

\section{A. Battery Management System}

The battery management system is the heart of the all battery monitoring activities. It manages the battery pack during charging and discharging activities. The main features of the BMS is to monitor battery internal temperature, internal cell voltage, internal pack voltage, internal cell current, internal pack current, $\mathrm{SoC}, \mathrm{SoH}$, SoF, and SoL in some cases, etc.

\section{B. Current Sensing operation}

A typical Electric vehicle uses a current sensor based on the principle of Hall Effect hence giving it the name hall effect sensor or hall sensor. The main function of the hall sensor are : over current fault detection, over current fault protection message to over current relay, current control for the motor and detection of the type of load. The current sensor generally works between the ranges of $0 \mathrm{~A}$ to $500 \mathrm{~A}$. (A refers to Ampere rating of current)

\section{Control unit of the Battery pack}

The battery pack is controlled by the battery pack electronic control unit. The ECU of the battery pack receives the data of all the high voltage components. The battery pack ECU interacts through all the other ECUs through the CAN bus, so the CAN bus acts as the medium of inter- communication of all ECUs. The contactors that allow the voltage to pass further get de energized if the voltage fails to fall between the range of $2.5 \mathrm{~V}$ and $4.5 \mathrm{~V}$. The voltage if not sensed to be in the range is detected by the BMS and the message appears on dashboard so as to indicate the driver. The current sensor data and fault signal data is also taken by the ECU to be displayed on the dashboard. In case the battery pack encounters any fault and if the fault is rectified internally, the vehicle has to be brought to a semi-permanent stand still so as to create an environment of safety for the vehicle and most importantly the driver and passengers as the fault signals encountered in the battery pack are of high current and high voltage and any small short circuit of lose wiring could be fatal.

\section{- MOTOR CONTROL SUBSYSTEM}

\section{A. Motor Controller}

The motor controllers' main function is to limit the current going to the motor and control the motors speed. The complexity of various technologies used in making the drive easy increases as the motor rating and capabilities increase. When the work load on a single ECU becomes more, the working tendency becomes complex and hence electric motor control units need to be integrated with the other electronic control units. The interaction between multiple control units is normally complex. The speed of the vehicle can normally range from 0 to an extensive throttle range which is determined by the torque and motor incoming currents which have to be balanced by power gate transistors so as to maintain an efficient operation and good speed range.

\section{B. Motor Controller ECU}

The motor controller works mainly because of the amount of throttle applied on the accelerator. The RPM is generated by the proximity sensor present in the pedal subsystem which implies that the pedal subsystem and motor control subsystem go hand in hand. The PWM signal varies according to the throttle applied on the pedal. The motor controller ECU is also responsible for the calculation of rpm produced and delivered to the wheels. The RPM is then calculated and transmitted to the dashboard ECU and the output appears in the dashboard in front of the driver. The front wheels have proximity sensors located in them which calculate the incoming speed on them and transmit the calculated value to dashboard ECU.

\section{- ACCELARATION \& BRAKE PEDAL SUBSYSTEM}

\section{A. Brake Over Travel Switch}

The Break Over Travel Switch (BOTS) is a safety switch installed in the brake pedal of the vehicle. The safety becomes active when the vehicle runs out of brake fluid or when the brake fluid mechanism fails. The BOTS gets pressed when the pedal becomes loose due to the lack of fluid and thereby triggering a safety mechanism which de energizes the main contactor relay that cuts the supply to the motor and the vehicle comes to a standstill.

\section{B. Accelerator \& Brake Pedal ECU}

The accelerator and brake pedal have a total of three potentiometers installed in them, two for the accelerator pedal and one for the brake pedal each of whose resistance measures up to a maximum of $5000 \mathrm{ohms}$. The value of the potentiometers depend upon the pressure applied by the pedals and the value is calculated using an analog to digital converter present in the pedal ECU. The two pedals have a tolerance of ten percent of the measured value. If the accelerator pedal value has a difference of over ten percent then a stop signal or a plausibility signal is sent to the motor controller. A stop signal is also sent in cases where the brake pedal exceeds twenty five percent and the accelerator pedal exceeds over twice that of brake plausibility signal, i.e. fifty percent. If the potentiometer 
gives a value of exactly 5 volts, it means that it is happening due to the action of the pull up resistor. This indicates that there is a floating signal in the output of the potentiometers which means that the output connects directly to a $5 \mathrm{~V}$ output of the safety system. The resistors range up to a hundred kilohms $(100 \mathrm{k} \Omega)$. The ECU waits for a plausibility to be rectified and in order to rectify, the driver must restart the vehicle, take it for service or in most cases there is auto plausibility reboot which rectifies the error and brings the condition to normal. If there is no plausibility then the average of the two throttle values must be calculated and sent to the motor controller and MC ECU via the controller area network bus.

\section{- DASHBOARD SUBSYSTEM}

\section{A. Dashboard}

The dashboard is the main display of the parameters calculated by various ECUs of the EV and is transmitted to the LED output display of the dashboard by the dashboard ECU via the CAN bus. The dashboard displays the following values:

$\begin{array}{ll}\text { i) } & \text { Battery temperature rise } \\ \text { ii) } & \text { Insulation leak or rupture } \\ \text { iii) } & \text { Voltage overshoot } \\ \text { iv) } & \text { Current overshoot } \\ \text { v) } & \text { Short circuit fault } \\ \text { vi) } & \text { Potentiometric fault in the pedal system }\end{array}$

\section{B. Control unit of the dashboard (ECU)}

One of the most important features of the control unit of the dashboard is to transmit the message to all ECUs that the vehicle is ready to start which is also known as the ready to drive state. The dashboard keeps getting updated according to the RPM reading it receives from the motor controller control unit via CAN bus. In a similar way the speedometer also gets updated. The Dashboard makes use of data acquisition in order to maintain and keep track of all the information it receives from all the ECUs, in this way there will be a log to see all previous values and information.

\section{INTEGRATED SYSTEM}

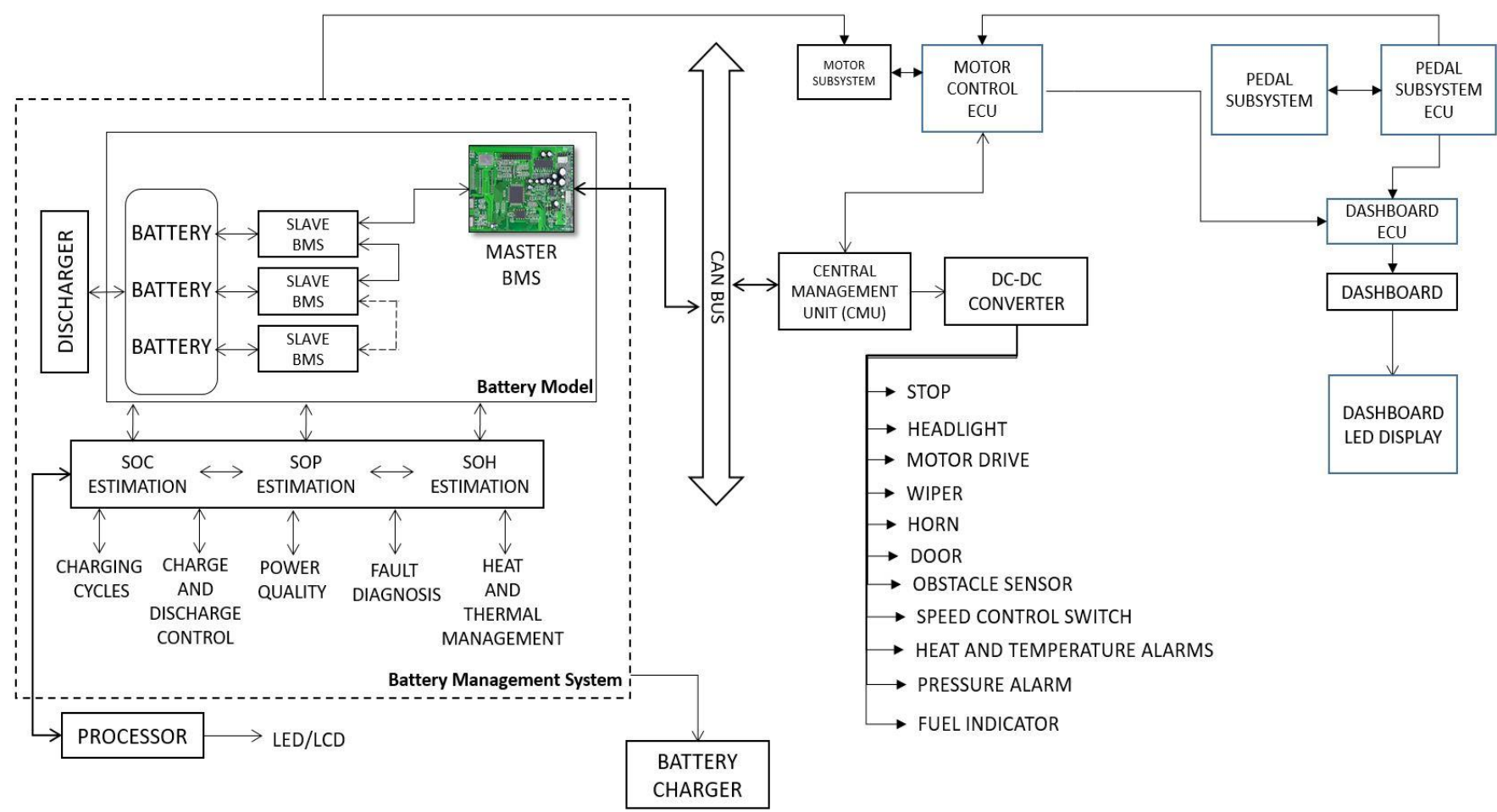

Fig.6 BMS integrated CAN bus control environment

The integrated system shown in the Fig.6, is the working control system of the electric vehicle. The CAN bus is the single serial bus communication medium connecting all the Electronic control units across the vehicle. As we know the BMS is the primary control unit for the battery pack of the vehicle that supplies the power to the motor for its running that ultimately produces torque and rpm for the wheels to rotate and thereby generating motion. The motor controller ECU is one of the most vital control units in the vehicle as it helps in the control of speed of the vehicle so its efficiency and working should be to the fullest of potential. The motor speed is converted to a digital value by the dashboard ECU 
and is displayed on the dashboard LED display. The CAN bus is responsible for all information and commands to be transmitted from one ECU to the other or from one subsystem to the other subsystem. The throttle subsystem more commonly known as the pedal subsystem is connected via CAN bus to the motor controller ECU and the dashboard ECU for the purpose of conveying fault or error signal to the dashboard display which will ultimately intimate the driver to stop the vehicle or restart the vehicle. The BMS is the heart for the safe and hassle-free running of the vehicle, the BMS decides the safety of car components and the safety of human driver in the car. The information coming from the BMS is converted to bits which can be interpreted by the CAN bus which can be further transferred to the dashboard subsystem and the motor control subsystem. The main information of the BMS that are converted to bits are the following:
i) State of Health (1 byte)
ii) State of Charge (1 byte)
iii) Thermal stability ( 2 bytes)
iv) Temperature (2 bytes)
v) Present battery pack voltage ( 2 bytes)
vi) Present battery pack current ( 2 bytes)
vii) Power remaining in the battery pack ( 2 bytes)

The working of multiple subsystems simultaneously causes the internals of the vehicle to heat up which may lead to short circuit, rupture of wiring or fires, this may be fatal to the vehicle and the human driver. In order to minimize the occurrence of such instances the thermal dissipation system comes into play. The thermal manager acts as the primary coolant for the internal components of the vehicle and is installed in places where there is transmission of high voltages and high currents. The sensor is connected to a cooling fan or an exhaust system and switches this system ON when the voltage in that particular system exceeds the threshold voltage limit. If the system does not cool even after the triggering of the exhaust system, then a message is sent via CAN bus to the dashboard display to intimate the human driver to switch the vehicle OFF. If the same system drops below a particular threshold voltage then the voltage sensor switches the system signal to a high resistance which will allow path for higher voltage. Once the voltage rating comes to the constant range, the signal switches to its normal path. Also in Fig.6, the BMS performs inter-cell charge balancing in $\mathrm{Li}$-ion and other closed-cell systems. The device is designed as a two-level control system which is divided as Battery Management Unit (BMU) which does the function of fan control, cell tapping or voltage extraction and monitoring of battery pack. The Central Management Unit (CMU) is a microprocessor that is interfaced to monitor the data limits along with a Printed Circuit Board (PCB) and sensors which shuts down the control circuitry when the limit is exceeded. The BMU is equipped with a Li-ion battery with a nominal voltage of 3.2 Volts for a stack of cells. It controls the voltage and temperature for the management of cells. The CMU operates the working of the battery to store data records through a communication interface via a CAN Bus. The CAN bus simplifies the circuit to integrate and enhance the communication using a transreciever with a DC-DC converter between the BMU and CMU for processing. The ECU in the BMS uses a DC-DC Converter and a voltage regulator to convert the $12 \mathrm{~V} \mathrm{DC}$ to $9 \mathrm{~V} \mathrm{DC}$, connected to a circuit that connects to all the sensors in an EV.

\section{SYSTEM FAULTS AND MITIGATION \\ i) Short circuit fault}

Short circuit faults occur when there is clustering of wiring in the system. When wires overlap over one another rupturing occur and thereby lead to short circuit faults between the metal casing and the exposed wire. In order to avoid the short circuit, a proper insulation must be done before installation of wires. The casing material must be made of a semi-conductive material that is resistive to high temperatures created during the short circuit faults.

\section{ii) Temperature instability}

Temperature instability leads to faulty operation of the battery management system. When there is a fluctuation in the temperature, the system goes out of its optimum working conditions and therefore loses on its performance. In order to mitigate this a proper thermal stability management and cooling system must be installed so as to maintain an optimum working temperature.

\section{iii) Acknowledgement error of CAN bus}

The acknowledgement error occurs in the CAN bus when the receiver fails to send an ACK message or the transmitter fails to see the ACK message sent by the receiver. This is usually detected by the bits, if it is 0 then it means the message has been conveyed to the receiver and 1 would indicate non-receipt and hence an error. When ACK message is not passed back to the transmitter, an error is signaled usually known as acknowledgement error. After this a frame is sent and the original message is sent once again to the receiver.

\section{iv) Cyclic redundancy check error in CAN bus}

Every message has to have the same value of CRC in the transmitting end and receiving end. The signal on reaching the receiving end is calculated and the CRC value is verified. If these values do not match, then the transmitting end receives a CRC error also known as Cyclic Redundancy error. An error message is generated in the next frame and the message in transmitted after an interval.

\section{v) Bit error of CAN bus}

A bit error occurs if there is a difference in the sending and receiving bit. The bit sent is usually assessed by the $\mathrm{CAN}$ bus and if there is found to a contrast in the 
sending and receiving bit then a bit error message is generated. Error stuffing or stuff error is a commonly used technique which purposefully generates a bit of a contrasting or opposite polarity. If there are more than a certain number of bits of the same polarity, then the CAN bus stuffs in a bit signal so as to help the receiver have a good synchronization. Say, if there are 4 bits of same polarity then one bit is stuffed in with an opposite polarity. If the same polarity bits exceed a number over six then the stuffed error process will not be valid as this will create an error signal and the bits have to be resent after an interval.

\section{vi) Form error of CAN bus}

It occurs when one or more dominant bits are detected in the four fixed and predetermined segments of CAN transmission. The four segments are end segment frame, inter segment frame, CRC delimiter and acknowledgement delimiter. After the error has occurred, the message is resent after an interval.

\section{FUTURE SCOPE AND APPLICATION}

Globally, Electric vehicles have revolutionized the automotive industry for the future. Slowly, it is pacing towards the next generation of mobility that has unveiled itself as a replacement for the traditional vehicles involving fuel such as petrol and diesel. Charging infrastructure and the battery performance is one of the key features of this development that substantially reduces the consumption of fuel proving to be environment friendly. Some of the applications of this interface in different areas include:

1. Aviation and Navigation

2. Escalators and Elevators

3. Agriculture

4. Medical Devices and instruments

5. Railways and Railroads

6. Power

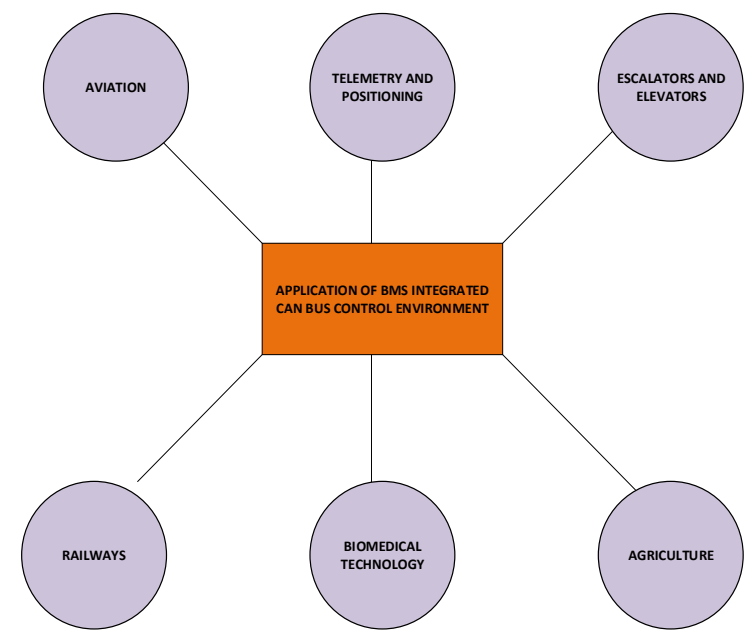

Fig.7 Applications of BMS integrated CAN bus control system

\section{CONCLUSION}

Electric vehicles are undoubtedly the future in the automotive industry because of its higher performance rate and safety improvements with low emission This paper presents how CAN bus can be interfaced with BMS to create an overall structure for real time control and reliable data communication. As we know, CAN Bus communication is indispensable when it comes to electric vehicle communication because of data transmission speed, reliability by reducing complication, accuracy in error detection along with lower hardware costs for multi signal processing techniques. This makes it ideal for implementation of CAN Bus in an Electric Vehicle for communication internally. By using this interface we can optimize the communication speed and fault/error detection that can be occurring at different points in the electric vehicle and can be effectively used to communicate to the driver. This paper has also reviewed the relevant data to form a complete idea of the integrated technology involved to interface CAN and BMS. To match the requirements it has compared many parameters and conducted diagnostic survey for accuracy to predict the vehicle safety to ensure a safer ride for the passengers onboard.

\section{REFERENCES}

[1] J. Kim, G.-S. Seo, C. Chun, B.-H. Cho, and S. Lee, 'OCV hysteresis effect-based SOC estimation in extended kalman filter algorithm for a LiFePO4/C cell," in Proc. IEEE International Electric Vehicle Conference, pp. 1-5, Mar. 2012.

[2] M. R. Vemparala, S. Yerabati, and G. I. Mary, "Performance analysis of controller area network based safety system in an electric vehicle," 2016 IEEE International Conference on Recent Trends in Electronics, Information \& Communication Technology (RTEICT), May 2016.

[3] L. Buccolini, A. Ricci, C. Scavongelli, G. DeMaso-Gentile, S Orcioni, and M. Conti, "Battery Management System (BMS) simulation environment for electric vehicles," 2016 IEEE 16th International Conference on Environment and Electrical Engineering (EEEIC), Jun. 2016.

[4] Zong-You Hou, Pang-Yen Lou, and C.-C. Wang, "State of charge, state of health, and state of function monitoring for EV BMS,' 2017 IEEE International Conference on Consumer Electronics (ICCE), 2017.

[5] S. S. Chethan, S. N. Kavya, M. Crasto, and P. G. Poddar, "Strategies for monitoring CAN node communication failures in the V2X eco-system," 2017 International Conference on Circuits, Controls, and Communications (CCUBE), Dec. 2017.

[6] A. Kurt, U. Dolu, B. Kaya, and G. Poyrazoglu, "Design of the Circuitry, Battery Management System, and Wireless Communication of an Electric Vehicle," 2019 1st Global Power, Energy and Communication Conference (GPECOM), Jun. 2019.

[7] D. D. Artakusuma, H. Afrisal, A. I. Cahyadi, and O. Wahyunggoro, "Battery management system via bus network for multi battery electric vehicle," 2014 International Conference on Electrical Engineering and Computer Science (ICEECS), Nov. 2014.

[8] C.-H. Hsu, K.-C. Chang, Y.-C. Ou, K.-Y. Liao, and C.-L. Wey, "On the implemenation of CAN buses to battery management systems," 2011 IEEE 54th International Midwest Symposium on Circuits and Systems (MWSCAS), Aug. 2011.

[9] B. O’Brien, "A battery management system for electric buses," Proceedings of 9th Annual Battery Conference on Applications and Advances.

[10] X. Jingjing and W. Guanghai, "Battery Management System of Electric Vehicle Based on CAN," 2015 International Conference on Intelligent Transportation, Big Data and Smart City, Dec. 2015

[11] Jinrui Nan, Li Zai, Zhifu Wang, and Jun Wang, "Bus Communication and Control Protocol Using the Electric Passenger 
Car Control System," 2006 6th World Congress on Intelligent Control and Automation, 2006.

[12] M. T. Lawder, B. Suthar, P. W. C. Northrop, S. De, C. M. Hoff, O. Leitermann, M. L. Crow, S. Santhanagopalan, and V. R. Subramanian, "Battery Energy Storage System (BESS) and Battery Management System (BMS) for Grid-Scale Applications," Proceedings of the IEEE, vol. 102, no. 6, pp. 1014-1030, Jun. 2014.

[13] Li Ran, Wu Junfeng, Wang Haiying, and Li Gechen, "Design method of CAN BUS network communication structure for electric vehicle," International Forum on Strategic Technology 2010, Oct. 2010.

[14] A. Zhang, S. Song, C. Wang, J. Zhang, K. Wang, and L. Li, "Research of battery management system for integrated power supply," 2017 Chinese Automation Congress (CAC), Oct. 2017.

[15] Wei Kong, "Integrated protection and control, network management - solution for DC traction power supply system," Eighth IEE International Conference on Developments in Power System Protection, 2004.

[16] Minxin Zheng, Bojin Qi, and Hongjie Wu, "A Li-ion battery management system based on CAN-bus for electric vehicle," 2008 3rd IEEE Conference on Industrial Electronics and Applications, Jun. 2008.

[17] K. M. S. KUMAR and B. G. KRISHNAN, "Design and Implementation of Vehicle Control System Using I2C Protocol," International Journal of Advanced Research in Electrical, Electronics and Instrumentation Engineering, vol. 3, no. 11, pp. 12933-12940, Nov. 2014.

[18] M. Brandl et al., "Batteries and battery management systems for electric vehicles," 2012 Design, Automation \& Test in Europe Conference \& Exhibition (DATE), Dresden, 2012, pp. 971-976, doi: 10.1109/DATE.2012.6176637

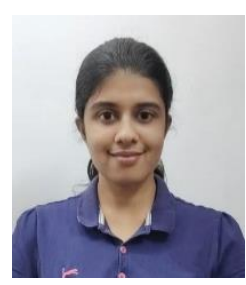

S.Divyashree, is a 3rd year Undergraduate Student. She is currently studying B.tech. ECE in SRM Institute of Science and Technology, Kattankulathur, India. Her research interests are Integrated circuits, electric vehicle and Renewable energy sources.

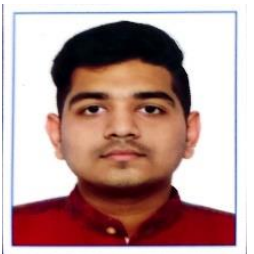

Prassanna Madhavan, is a $3^{\text {rd }}$ year Undergraduate Student. He is currently studying B.tech. EEE in SRM Institute of Science and Technology, Kattankulathur, India. His research interests are power electronics, electric vehicle and Renewable energy sources.

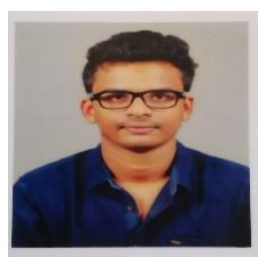

A.Ranjeev, is a $3^{\text {rd }}$ year Undergraduate Student. He is currently studying B.tech. EEE in SRM Institute of Science and Technology, Kattankulathur, India. His research interests are power electronics, electric vehicle and electrical machines. 